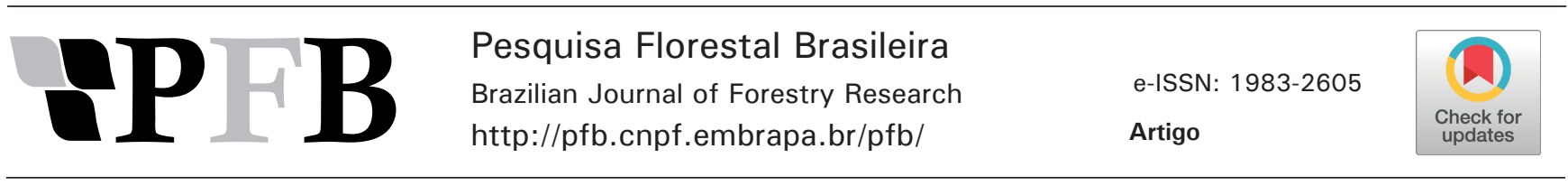

\title{
Análise comparativa de dois remanescentes arbustivo-arbóreo de Caatinga
}

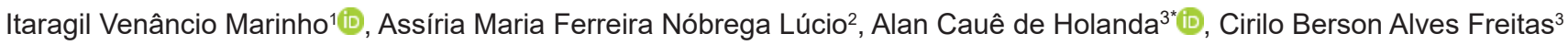 \\ ${ }^{1}$ Empaer, Rodovia BR 230, km 13,3, S/N, Parque Esperança, LTO Morada Nova, L-26, CEP 58108-502, Cabedelo, PB, Brasil \\ ¿Universidade Federal de Campina Grande, Avenida Universitária, s/n, CP. 61, Santa Cecília, CEP 58708-110, Patos, PB, Brasil \\ ${ }^{3}$ Universidade Federal Rural do Semi-Árido, Departamento de Ciências Agronômicas e Florestais, Avenida Francisco Mota, nº 572 , Costa e Silva, CEP 59625- \\ 900, Mossoró, RN, Brasil
}

\section{"Autor correspondente:}

alan.holanda@ufersa.edu.br

Termos para indexação:

Fitossociologia

Diversidade biológica

Regeneração natural

Index terms:

Phytosociology

Biological diversity

Natural regeneration

Histórico do artigo:

Recebido em 20/10/2017

Aprovado em 03/05/2019

Publicado em 31/12/2019

\section{cc) $\$$

BY NC ND

Resumo - O objetivo desse trabalho foi analisar se remanescentes de Caatinga arbustivoarbórea com distintas composições florísticas possuem similaridades fitoecológicas. Foram estudadas duas áreas: uma localizada no Seridó Potiguar (Barrentas), e outra no Cariri Paraibano (Passagem Rasa). Através de amostragem aleatória por parcelas fixas, foram mensurados árvores e arbustos do estrato florestal e analisada a composição florística e diversidade. Em Barrentas foram amostrados, no estrato adulto, 1.418 indivíduos, distribuídos em cinco famílias, 11 gêneros e 11 espécies, com uma densidade de 5.300 fustes ha $^{-1}$ e em Passagem Rasa, 1.565 indivíduos, representando 10 famílias, 20 gêneros e 26 espécies, com 4.175 fustes ha ${ }^{-1}$. Na regeneração natural, foram inventariados 398 indivíduos de cinco famílias, 10 gêneros e 12 espécies em Barrentas e 680 indivíduos, de cinco famílias, 10 gêneros e 11 espécies em Passagem Rasa. As áreas inventariadas apresentam baixa similaridade florística e estruturais para as variáveis densidade e dominância. Em principio, isso ocorre por maior influência das condições edafoclimáticas associadas a fatores antrópicos.

\section{Comparative analysis of two shrub remnants of Caatinga}

Abstract - The objective of this work was to analyze whether remnants of shrub wooded Caatinga with different floristic composition present phytoecological similarities. The study was carried out in two areas: in Seridó Potiguar (Barrentas) and in Cariri Paraibano (Passagem Rasa). We used ramdomly located sample plots for adult tree and regenerating. Forest individuals were measured, and the composition and floristic diversity were analyzed. We found 1,418 individuals in the adult trees stratum distributed into five families, 11 genres and 11 forest species with a density of 5,300 stem ha-1 in Barrentas and 1,565 individuals distributed into 10 families, 20 genres and 26 species

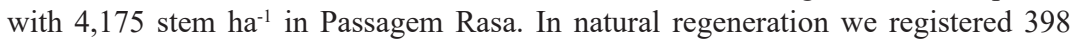
individuals, of five families, 10 genres and 12 species in Barrentas and 680 individuals distributed into five families, 10 genres and 11 species in Passagem Rasa. The studied areas presented low floristic and structural similarity, considering the variables density and dominance. In principle, this is due to the greater influence of the edaphoclimatic conditions associated with anthropic factors. 


\section{Introdução}

A exploração desordenada do bioma Caatinga tem levado à uma baixa diversidade florística do componente arbustivo-arbóreo (Fabricante \& Andrade, 2007) e consequente degradação ambiental (Barbosa et al., 2007; Cunha et al., 2008). Mesmo assim, a Caatinga tem um alto poder de resiliência, podendo recuperar sua diversidade florística após abandono das atividades antrópicas, como observaram Andrade et al. (2009).

Embora estudos tenham sido realizados para ampliar o conhecimento sobre a dinâmica e estrutura florestal, análises comparativas ainda são insuficientes para explicar as relações estruturais em remanescentes de Caatinga, pois mesmo sendo encontrada homogeneidade quanto ao estado da vegetação em áreas distintas (Oliveira et al., 2009), diferenças na composição florística e riqueza de espécies foram constatadas quando analisadas áreas em diferentes tipos de solo (Pinheiro et al., 2010), e sob diferentes altitudes (Ferraz et al., 1998). Outros estudos revelaram não ocorrer diferenças na riqueza e diversidade de espécies entre ambientes florestais, embora a composição florística mostre o contrário (Rabelo et al., 2015).

Nesta perspectiva, este trabalho objetivou analisar a hipótese de que remanescentes de Caatinga arbustivoarbórea, com histórico de perturbações similares, porém separados geograficamente e sob condições edafoclimáticas diferentes, se distinguem em termos de composição florística e estrutural entre os indivíduos lenhosos, sendo utilizados dois remanescentes para área de estudo, um no Seridó Potiguar (Barrentas), e outro no Cariri Paraibano (Passagem Rasa).

\section{Material e métodos}

Os remanescentes de Caatinga estudados situam-se em imóveis rurais em Sumé, PB (Passagem Rasa, com 166,42 ha), e em Acari, RN (Barrentas, com 129,07 ha).

Passagem Rasa (7³4'30”' S e 36 53'20” W) está localizada na microrregião do Cariri Paraibano, no Planalto da Borborema (IBGE, 2006), com altitudes variando em torno dos $600 \mathrm{~m}$ (relevo plano a suaveondulado e ondulado), com Neossolos Litólicos, textura arenosa e/ou média, fase pedregosa e rochosa e Luvissolos Crômicos (Sudema, 2004). O clima da região é Tropical da Zona Equatorial (semiárido quente), com até 11 meses de seca, e média pluviométrica anual variando em torno dos $500 \mathrm{~mm}$, temperaturas médias máximas em torno dos $25{ }^{\circ} \mathrm{C}$ e as médias mínimas, dos $21^{\circ} \mathrm{C}$ (IBGE, 2002; Sudema, 2004). A vegetação regional compreende a Savana Estépica Arborizada (IBGE, 2004).

Barrentas (6²0’30” S e 36²' 30” W) está localizada na mesorregião Central Potiguar, microrregião do Seridó Oriental, na Depressão Sertaneja, de relevo plano a suave-ondulado, altitudes variando entre $250 \mathrm{~m}$ a $300 \mathrm{~m}$, e ondulações formando vales em "V" abertos, de Luvissolos Crômicos, rasos, com fases pedregosas (horizontes cascalhentos), susceptíveis à erosão e com bom nível de drenagem, ricos em nutrientes, mas com uso restrito para agricultura (IBGE, 2006; Bezerra Júnior \& Silva, 2007). O clima da região é Tropical da Zona Equatorial (quente e semiárido), médias pluviométricas anuais entre $400 \mathrm{~mm}$ a $600 \mathrm{~mm}$, com período seco de 7 a 8 meses e temperaturas médias quentes, maiores que 18 ${ }^{\circ} \mathrm{C}$ em todos os meses do ano, médias, das máximas, em torno dos $33{ }^{\circ} \mathrm{C}$ e, das mínimas, de $22{ }^{\circ} \mathrm{C}$ (IBGE, 2002; Ana, 2005). A vegetação regional apresenta acentuado nível de endemismo, sendo caracterizada como Savana Estépica Arborizada, com estratos arbóreo (de árvores baixas providas de acúleos ou espinhos) e gramíneolenhoso sazonal, com diversas cactáceas e bromélias (IBGE, 2004).

Nas duas áreas de estudo (Passagem Rasa, ha mais de 20 anos e Barrentas, há mais de 30 anos) a vegetação nativa vinha sendo explorada para pastejo de animais de criação e retirada esporádica de produtos madeireiros (estacas e mourões para cercas).

A amostragem da vegetação foi realizada em parcelas quadradas de $400 \mathrm{~m}^{2}$ alocadas aleatoriamente, conforme recomendações da Rede de Manejo Florestal da Caatinga (2005), sendo 10 parcelas em Barrentas, e 12 parcelas em Passagem Rasa. No interior das parcelas amostrais, na extremidade inferior à direita, sentido norte-sul, foi lançada uma subparcela quadrada de $25 \mathrm{~m}^{2}$, para avaliação da regeneração natural.

Foram considerados adultos os indivíduos lenhosos do estrato arbustivo e arbóreo com circunferência a 1,30 m da superfície do solo (CAP) $\geq 6 \mathrm{~cm}$, sendo considerados regenerantes os indivíduos com $\mathrm{CAP}<6 \mathrm{~cm}$, qualificados segundo as classes de altura $(\mathrm{H})$ estabelecidas, em que $\mathrm{C} 1=0,5 \mathrm{~m} \leq \mathrm{H} \leq 1,0 \mathrm{~m}$ e $\mathrm{C} 2=\mathrm{H}>1 \mathrm{~m}$. Foram incluídas no levantamento as espécies de cactos presentes nas parcelas amostrais.

As espécies foram reconhecidas in loco pelo registro dos nomes populares, com coleta de material botânico para herborização e posterior identificação e confirmação 
dos nomes científicos, realizada por comparações com material depositado no Herbário da Universidade Federal de Campina Grande, Campus de Patos, PB (onde o material herborizado foi depositado), consultas à literatura especializada (Siqueira Filho et al., 2009; Gamarra-Rojas et al., 2010; Forzza et al., 2010; Silva et al., 2010) e a especialistas. Foi adotado o sistema de classificação The Angiosperm Phylogeny Group (APG, 2009), incluindo informações sobre o hábito e status de conservação das espécies (Brasil, 2008).

A suficiência amostral foi verificada pela curva espécie $\mathrm{x}$ área. Foram calculados para os indivíduos adultos os parâmetros fitossociológicos da estrutura horizontal, que incluíram a densidade absoluta e relativa, frequência relativa, dominância relativa e valor de importância e, para a regeneração natural, as densidades relativas nas diferentes classes de altura (Felfili \& Rezende, 2003), com auxílio do programa computacional MATA NATIVA 2, versão 2.10 (Cientec, 2006).

A similaridade entre as áreas estudadas foi verificada pela aplicação do teste de similaridade entre comunidades de Jaccard (Cientec, 2006). A diversidade florística para os indivíduos adultos e regenerantes foi calculada com base na distribuição dos indivíduos em espécies e famílias e nos índices de diversidade ecológica de Shannon-Weaver $\left(H^{\prime}\right)$, equabilidade de Pielou $\left(J^{\prime}\right)$ (Brower \& Zarr, 1984; Magurran, 1988). As médias dos índices de diversidade, para as duas áreas de estudo, foram comparadas pelo teste de Tukey, com uso do programa computacional de assistência estatística Assistat, versão beta 7.6 (Silva \& Azevedo, 2010).

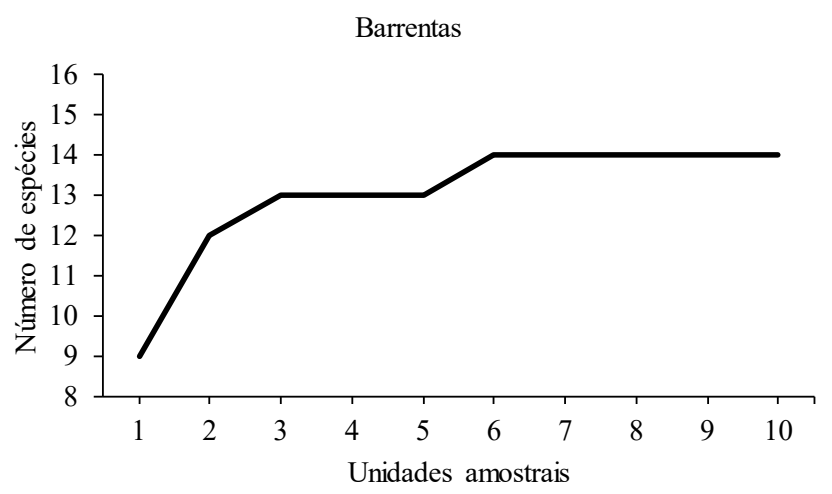

\section{Resultados}

O platô formado nas curvas espécie $\mathrm{x}$ área (Figura 1) demonstra que o levantamento realizado nas áreas de estudo foi satisfatório, indicando que a área amostrada em Barrentas $\left(4.000 \mathrm{~m}^{2}\right)$ e em Passagem Rasa $\left(4.800 \mathrm{~m}^{2}\right)$ foi suficiente para representar a quantidade de espécies florestais.

Observou-se a estabilização das curvas espécie x área para os dois remanescentes (Figura 1), indicando que a área mínima representativa floristicamente foi atingida, ou seja, o número de parcelas inventariadas foi suficiente para representar florísticamente as respectivas áreas.

Foram inventariados em Barrentas 1.418 indivíduos, pertencentes a cinco famílias, 11 gêneros e 11 espécies identificadas. Passagem Rasa foi representada por 1.565 indivíduos, distribuídos em 10 famílias, 20 gêneros e 26 espécies, com uma espécie desconhecida e duas indeterminadas (Tabela 1).

Em Barrentas foi amostrado apenas um indivíduo das espécies Anadenanthera colubrina e Spondias tuberosa e em Passagem Rasa, apenas um individuo de Cordia leucocephala, Capparis yco, Mimosa tenuiflora, Schinopsis brasiliensis e Senna macranthera.

Em Barrentas, as famílias Fabaceae e Euphorbiaceae foram representadas por cinco e três espécies respectivamente. Em Passagem Rasa, Fabaceae foi representada por oito espécies, Euphorbiaceae, por sete e Anacardiaceae, por duas. As demais famílias tinham apenas uma espécie nas duas áreas de estudo (Figura 2).

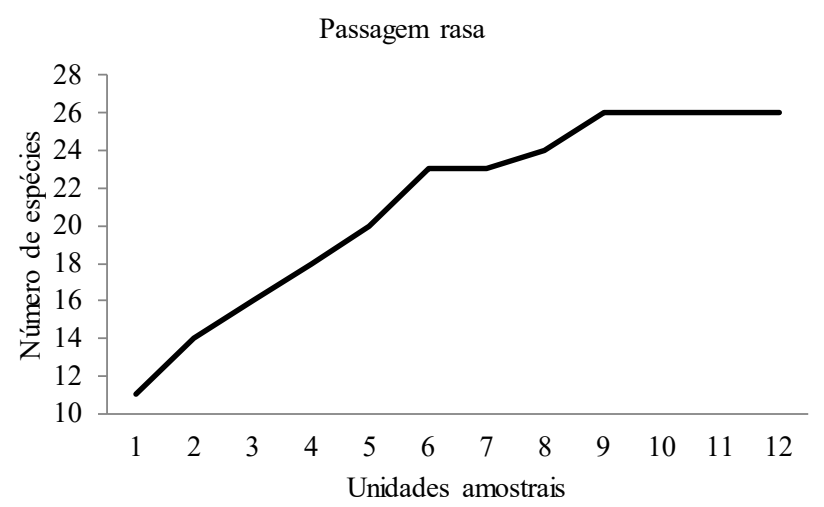

Figura 1. Curva espécie $x$ área para a amostragem da vegetação arbustivo-arbórea nas áreas estudadas nos estados do Rio Grande do Norte e Paraíba.

Figure 1. Curve species $x$ area for sampling shrub wooded species in the studied areas in the states of Rio Grande do Norte and Paraíba. 
Tabela 1. Relação florística das famílias/espécies inventariadas em remanescentes de Caatinga localizadas em Barrentas (B) no Rio Grande do Norte e Passagem Rasa (PR) na Paraíba, com os respectivos hábitos e estatos de conservação.

Table 1. Floristic of families/species inventoried in Caatinga remnants located in Barrentas (B), Rio Grande do Norte State and in Passagem Rasa (PR), Paraíba, State with their respective habit and conservation status.

\begin{tabular}{|c|c|c|c|c|}
\hline \multirow{2}{*}{ Famílias/espécies } & \multicolumn{2}{|c|}{ Local } & \multirow{2}{*}{ Hábito } & \multirow{2}{*}{ Conservação } \\
\hline & B & PR & & \\
\hline \multicolumn{5}{|l|}{ Anacardiaceae } \\
\hline Myracrodruon urundeuva M. Allemao & - & $\mathrm{X}$ & A & $\mathrm{S}^{1}$ \\
\hline Schinopsis brasiliensis Engl. var. brasiliensis & - & $\mathrm{X}$ & A & $\mathrm{S}^{1}$ \\
\hline Spondias tuberosa Arruda & $\mathrm{X}$ & - & A & $\mathrm{N}$ \\
\hline \multicolumn{5}{|l|}{ Apocynaceae } \\
\hline Aspidosperma pyrifolium Mart. & $\mathrm{X}$ & $\mathrm{X}$ & A & $\mathrm{N}$ \\
\hline \multicolumn{5}{|l|}{ Boraginaceae } \\
\hline Cordia leucocephala Moric. & - & $\mathrm{X}$ & $\mathrm{Ab}$ & $\mathrm{N}$ \\
\hline \multicolumn{5}{|l|}{ Burseraceae } \\
\hline Commiphora leptophloeos (Mart.) J. B. Gillett & $\mathrm{X}$ & $\mathrm{X}$ & A & $\mathrm{N}$ \\
\hline \multicolumn{5}{|l|}{ Capparaceae } \\
\hline Capparis yco (Mart.) Eichler & - & $\mathrm{X}$ & A & $\mathrm{N}$ \\
\hline \multicolumn{5}{|l|}{ Combretaceae } \\
\hline Combretum leprosum Mart. & - & $\mathrm{X}$ & $\mathrm{Ab}$ & $\mathrm{N}$ \\
\hline \multicolumn{5}{|l|}{ Euphorbiaceae } \\
\hline Acalypha multicaulis Müll. Arg. & - & $\mathrm{X}$ & $\mathrm{Ab}$ & $\mathrm{N}$ \\
\hline Cnidoscolus quercifolius Pohl & $\mathrm{X}$ & - & A & $\mathrm{N}$ \\
\hline Croton sp. & - & $\mathrm{X}$ & $\mathrm{Ab}$ & $\mathrm{N}$ \\
\hline Croton argyrophyllus Kunth. in Humb. & - & $\mathrm{X}$ & $\mathrm{Ab}$ & $\mathrm{N}$ \\
\hline Croton blanchetianus Baill. & $\mathrm{X}$ & $\mathrm{X}$ & $\mathrm{Ab}$ & $\mathrm{N}$ \\
\hline Jatropha mollissima (Pohl) Baill. & $\mathrm{X}$ & $\mathrm{X}$ & $\mathrm{Ab}$ & $\mathrm{N}$ \\
\hline Manihot glaziovii Müll. Arg. & - & $\mathrm{X}$ & A & $\mathrm{N}$ \\
\hline Sapium glandulosum (L.) Morong & - & $\mathrm{X}$ & A & $\mathrm{N}$ \\
\hline \multicolumn{5}{|l|}{ Fabaceae } \\
\hline Amburana cearensis (Allemão) A. C. Sm. & - & $\mathrm{X}$ & A & $\mathrm{N}^{2}$ \\
\hline Anadenanthera colubrina (Vell.) Brenan var. cebil (Griseb.) Altschul & $\mathrm{X}$ & - & A & $\mathrm{N}$ \\
\hline Bauhinia cheilantha (Bong.) D. Dietr. & - & $\mathrm{X}$ & A & $\mathrm{N}$ \\
\hline Mimosa adenophylla Taub. var. mitis Barneby & $\mathrm{X}$ & - & $\mathrm{Ab}$ & $\mathrm{N}$ \\
\hline Mimosa ophthalmocentra Mart. ex Benth. & - & $\mathrm{X}$ & $\mathrm{Ab}$ & $\mathrm{N}$ \\
\hline Mimosa tenuiflora (Willd.) Poir. & $\mathrm{X}$ & $\mathrm{X}$ & A & $\mathrm{N}$ \\
\hline Parapiptadenia zehntneri (Harms) M. P. Lima \& H. C. Lima & - & $\mathrm{X}$ & A & $\mathrm{N}$ \\
\hline Piptadenia stipulacea (Benth.) Ducke & $\mathrm{X}$ & $\mathrm{X}$ & A & $\mathrm{N}$ \\
\hline Poincianella pyramidalis (Tul.) L. P. Queiroz & $\mathrm{X}$ & $\mathrm{X}$ & A & $\mathrm{N}$ \\
\hline Senna macranthera (Collad.) H. S. Irwin \& Barneby var. macranthera & - & $\mathrm{X}$ & A & $\mathrm{N}$ \\
\hline \multicolumn{5}{|l|}{ Lauraceae } \\
\hline Endlicheria paniculata (Spreng.) J. F. Macbr. & - & $\mathrm{X}$ & $\mathrm{Ab}$ & $\mathrm{N}$ \\
\hline \multicolumn{5}{|l|}{ Malpighiaceae } \\
\hline Desconhecida & - & $\mathrm{X}$ & $\mathrm{Ab}$ & - \\
\hline \multicolumn{5}{|l|}{ Família Indeterminada } \\
\hline Indeterminada 1 & - & $\mathrm{X}$ & A & - \\
\hline Indeterminada 2 & - & $\mathrm{X}$ & A & - \\
\hline
\end{tabular}

Em que: $\mathrm{A}=$ arbóreo; $\mathrm{Ab}$ = arbustivo; $\mathrm{N}$ = não ameaçada; $\mathrm{S}$ = ameaçada. Nota 1: Status de conservação segundo a lista do Anexo I de Brasil (2008). Nota 2: Embora presente na lista do Anexo I de Brasil (2008) com status de ameaçada de extinção, foi indicado para o bioma Amazônia. 

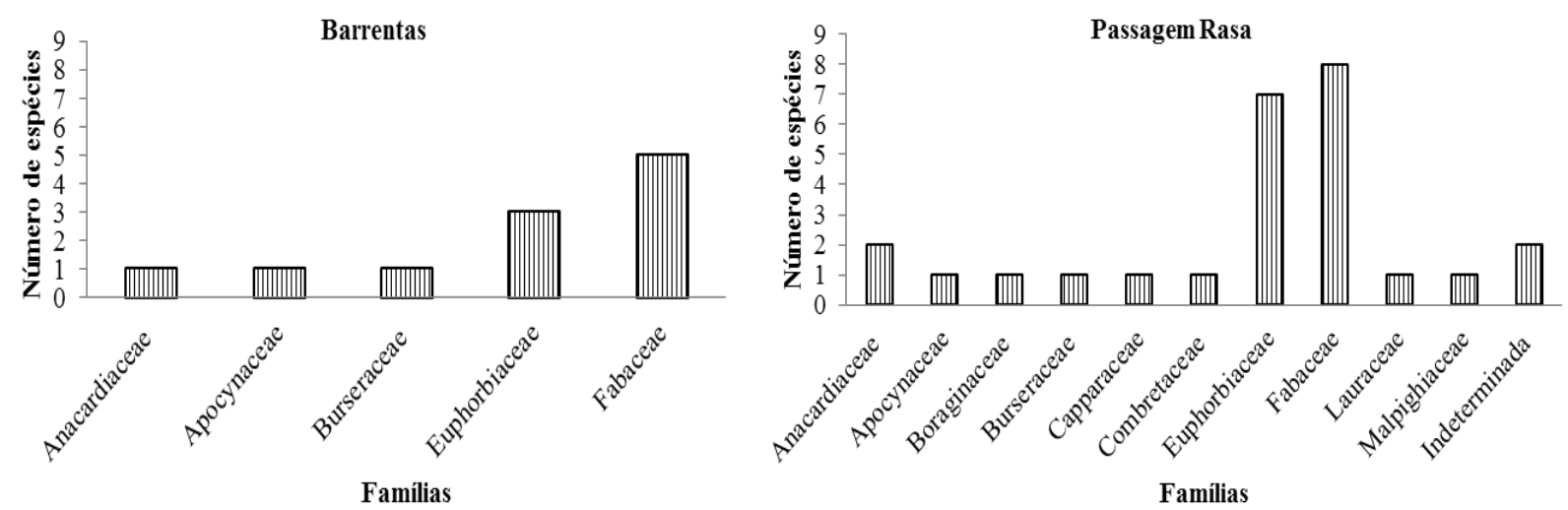

Figura 2. Número de espécies por famílias em remanescentes de Caatinga localizadas em Barrentas, RN e Passagem Rasa, PB Figure 2. Number of species by families in Caatinga remnants located in Barrentas, RN and Passagem Rasa, PB.

Foi estimada uma densidade de 3.545 ind $\mathrm{ha}^{-1} \mathrm{em}$ Barrentas e de 3.260 ind ha ${ }^{-1}$ em Passagem Rasa (Tabela 2). As espécies Poincianella pyramidalis, Mimosa tenuiflora, Croton blanchetianus e Aspidosperma pyrifolium apresentaram os maiores valores de importância (VI) em Barrentas (totalizando 77\%). Em Passagem Rasa as espécies com maior VI foram Poincianella pyramidalis, Croton blanchetianus, Acalypha multicaulis e Aspidosperma pyrifolium (representando 65,7\%).

A regeneração natural em Barrentas foi representada por 398 indivíduos, pertencentes a cinco famílias, 10 gêneros, e 12 espécies. Em Passagem Rasa, foram 680 indivíduos distribuídos em cinco famílias, 10 gêneros, e 11 espécies (Tabela 3).

As famílias Euphorbiaceae e Fabaceae foram as mais representativas do estrato regenerante nas duas áreas, assim como para o estrato de árvores adultas.

Em Barrentas, o estrato regenerante tinha mais espécies (12) que o estrato adulto (11), com diminuição de um gênero (de 11 para 10). Em Passagem Rasa a diminuição foi ainda maior, de 10 para cinco famílias, de 20 para 10 gêneros, e de 26 para 11 espécies. Nenhuma das espécies amostradas na regeneração natural encontra-se com status de ameaçada de extinção.
Quando se compara a vegetação do extrato adulto e regenerante (Tabelas 1 e 3), observa-se que em Barrentas, Anadenanthera colubrina, Commiphora leptophloeos e Spondias tuberosa, inventariadas no estrato adulto, não foram amostradas no estrato regenerante. Em Passagem Rasa, Amburana cearensis, Capparis yco, Combretum leprosum, Croton argyrophyllus, Manihot glaziovii, Myracrodruon urundeuva, Parapiptadenia zehntneri, Schinopsis brasiliensis, Senna macranthera e as espécies desconhecidas/indeterminadas, presentes no estrato de árvores adultas, não foram amostradas no estrato regenerante. Em ambas as áreas, Commiphora leptophloeos, inventariada no estrato adulto, não foi inventariada no estrato regenerante. Aspidosperma pyrifolium, Croton blanchetianus, Jatropha molissima e Poincianella pyramidalis aparecem nos dois estratos (adulto e regenerante).

Os índices de diversidade e equabilidade para o estrato regenerante em Barrentas $\left(H^{\prime}\right.$ de 1,14 e $J^{\prime}$ de 0,77$)$ e Passagem Rasa $\left(H^{\prime}\right.$ de 1,18 e $J^{\prime}$ de 0,84$)$ demonstram similaridade entre as mesmas, não havendo diferenças estatísticas entre as médias dos índices estimados.

Para o estrato regenerante em Barrentas, foi estimada uma densidade de 15.920 ind $\mathrm{ha}^{-1}$, enquanto que em Passagem Rasa a densidade foi estimada em 22.667 ind ha $^{-1}$,com maior regeneração natural de Croton blanchetianus (Tabela 4). 
Tabela 2. Estrutura horizontal do estrato adulto dos indivíduos inventariados em remanescentes de Caatinga localizadas em Barrentas (B) no Rio Grande do Norte e Passagem Rasa (PR) na Paraíba.

Table 2. Horizontal structure of the adult stratum of the individuals inventoried in Caatinga remnant located in Barrentas (B) in Rio Grande do Norte State and Passagem Rasa (PR) in Paraíba State.

\begin{tabular}{|c|c|c|c|c|c|c|c|c|}
\hline \multirow{2}{*}{ Espécies } & \multicolumn{2}{|c|}{ DA } & \multicolumn{2}{|c|}{ DR } & \multicolumn{2}{|c|}{ FR } & \multicolumn{2}{|c|}{ DoR } \\
\hline & B & PR & B & PR & B & PR & B & PR \\
\hline Acalypha multicaulis & - & 808 & - & 24,79 & - & 8,33 & - & 4,60 \\
\hline Amburana cearensis & - & 8 & - & 0,26 & - & 2,50 & - & 2,25 \\
\hline Anadenanthera colubrina & 3 & - & 0,07 & - & 1,47 & - & 0,02 & - \\
\hline Aspidosperma pyrifolium & 485 & 194 & 13,68 & 5,94 & 14,71 & 9,17 & 8,47 & 9,27 \\
\hline Bauhinia cheilantha & - & 48 & - & 1,47 & - & 6,67 & - & 1,08 \\
\hline Capparis yco & - & 2 & - & 0,06 & - & 0,83 & - & 0,43 \\
\hline Cnidoscolus quercifolius & 30 & - & 0,85 & - & 4,41 & - & 6,57 & - \\
\hline Combretum leprosum & - & 6 & - & 0,19 & - & 1,67 & - & 0,02 \\
\hline Commiphora leptophloeos & 40 & 13 & 1,13 & 0,38 & 10,29 & 2,50 & 3,36 & 5,44 \\
\hline Cordia leucocephala & - & 2 & - & 0,06 & - & 0,83 & - & 0,00 \\
\hline Croton sp. & - & 25 & - & 0,77 & - & 2,50 & - & 0,08 \\
\hline Croton argyrophyllus & - & 13 & - & 0,38 & - & 0,83 & - & 0,05 \\
\hline Croton blanchetianus & 773 & 1079 & 21,79 & 33,10 & 14,71 & 9,17 & 5,09 & 5,91 \\
\hline Endlicheria paniculata & - & 35 & - & 1,09 & - & 1,67 & - & 0,22 \\
\hline Jatropha mollissima & 55 & 90 & 01,55 & 2,75 & 11,76 & 9,17 & 1,25 & 0,89 \\
\hline Manihot glaziovii & - & 23 & - & 0,70 & - & 5,00 & - & 1,18 \\
\hline Mimosa adenophylla & 8 & - & 0,21 & - & 1,47 & - & 0,04 & - \\
\hline Mimosa ophthalmocentra & - & 85 & - & 2,62 & - & 9,17 & - & 3,21 \\
\hline Mimosa tenuiflora & 535 & 2 & 15,09 & 0,06 & 13,24 & 0,83 & 21,52 & 0,27 \\
\hline Myracrodruon urundeuva & - & 38 & - & 1,15 & - & 5,83 & - & 5,14 \\
\hline Parapiptadenia zehntneri & - & 21 & - & 0,64 & - & 4,17 & - & 2,29 \\
\hline Piptadenia stipulacea & 188 & 13 & 5,29 & 0,38 & 11,76 & 2,50 & 3,27 & 0,57 \\
\hline Poincianella pyramidalis & 1428 & 733 & 40,27 & 22,49 & 14,71 & 10,00 & 47,64 & 54,40 \\
\hline Sapium glandulosum & - & 13 & - & 0,38 & - & 2,50 & - & 0,48 \\
\hline Schinopsis brasiliensis & - & 2 & - & 0,06 & - & 0,83 & - & 2,11 \\
\hline Senna macranthera & - & 2 & - & 0,06 & - & 0,83 & - & 0,02 \\
\hline Spondias tuberosa & 3 & - & 0,07 & - & 01,47 & - & 2,77 & - \\
\hline Desconhecida & - & 2 & - & 0,06 & - & 0,83 & - & 0,02 \\
\hline Indeterminada 1 & - & 2 & - & 0,06 & - & 0,83 & - & 0,02 \\
\hline Indeterminada 2 & - & 2 & - & 0,06 & - & 0,83 & - & 0,02 \\
\hline
\end{tabular}

Em que: $\mathrm{DA}=$ densidade absoluta; $\mathrm{DR}=$ densidade relativa; $\mathrm{FR}=$ frequência relativa e $\mathrm{DoR}=$ dominância relativa. 
Tabela 3. Relação florística de famílias/espécies inventariadas nas subparcelas de regeneração natural em Barrentas (B) no Rio Grande do Norte e Passagem Rasa (PR) na Paraíba com os respectivos hábitos e estatos de conservação.

Table 3. Floristic relationship of families / species inventoried in the natural regeneration subplots in Barrentas (B), Rio Grande do Norte State and in Passagem Rasa (PR), Paraíba State with their respective habit and conservation status.

\begin{tabular}{|c|c|c|c|c|}
\hline \multirow{2}{*}{ Famílias/espécies } & \multicolumn{2}{|c|}{ Local } & \multirow{2}{*}{ Hábito } & \multirow{2}{*}{ Status } \\
\hline & B & PR & & \\
\hline \multicolumn{5}{|l|}{ Apocynaceae } \\
\hline Aspidosperma pyrifolium Mart. & $\mathrm{X}$ & $\mathrm{X}$ & A & $\mathrm{N}$ \\
\hline \multicolumn{5}{|l|}{ Boraginaceae } \\
\hline Cordia leucocephala Moric. & - & $\mathrm{X}$ & $\mathrm{Ab}$ & $\mathrm{N}$ \\
\hline \multicolumn{5}{|l|}{ Cactaceae } \\
\hline $\begin{array}{l}\text { Pilosocereus gounellei (F. A. C. } \\
\text { Weber) Byles \& G. D. Rowley } \\
\text { subsp. gounellei }\end{array}$ & $\mathrm{X}$ & - & $\mathrm{C}$ & $\mathrm{N}$ \\
\hline \multicolumn{5}{|l|}{ Euphorbiaceae } \\
\hline Acalypha multicaulis Müll. Arg. & - & $\mathrm{X}$ & $\mathrm{Ab}$ & $\mathrm{N}$ \\
\hline Cnidoscolus quercifolius Pohl & $\mathrm{X}$ & - & A & $\mathrm{N}$ \\
\hline Croton sp. & - & $\mathrm{X}$ & $\mathrm{Ab}$ & $\mathrm{N}$ \\
\hline Croton blanchetianus Baill. & $\mathrm{X}$ & $\mathrm{X}$ & $\mathrm{Ab}$ & $\mathrm{N}$ \\
\hline Croton campestris A.St.-Hil. & $\mathrm{X}$ & - & $\mathrm{Ab}$ & $\mathrm{N}$ \\
\hline Jatropha mollissima (Pohl) Baill. & $\mathrm{X}$ & $\mathrm{X}$ & $\mathrm{Ab}$ & $\mathrm{N}$ \\
\hline Sapium glandulosum (L.) Morong & - & $\mathrm{X}$ & A & $\mathrm{N}$ \\
\hline \multicolumn{5}{|l|}{ Fabaceae } \\
\hline $\begin{array}{l}\text { Bauhinia cheilantha (Bong.) D. } \\
\text { Dietr. }\end{array}$ & - & $\mathrm{X}$ & A & $\mathrm{N}$ \\
\hline $\begin{array}{l}\text { Mimosa adenophylla Taub. var. } \\
\text { mitis Barneby }\end{array}$ & $\mathrm{X}$ & - & $\mathrm{Ab}$ & $\mathrm{N}$ \\
\hline $\begin{array}{l}\text { Mimosa ophthalmocentra Mart. ex } \\
\text { Benth. }\end{array}$ & - & $\mathrm{X}$ & $\mathrm{Ab}$ & $\mathrm{N}$ \\
\hline Mimosa tenuiflora (Willd.) Poir. & $\mathrm{X}$ & - & A & $\mathrm{N}$ \\
\hline $\begin{array}{l}\text { Piptadenia stipulacea (Benth.) } \\
\text { Ducke }\end{array}$ & $\mathrm{X}$ & - & A & $\mathrm{N}$ \\
\hline $\begin{array}{l}\text { Poincianella pyramidalis (Tul.) L. } \\
\text { P. Queiroz }\end{array}$ & $\mathrm{X}$ & $\mathrm{X}$ & A & $\mathrm{N}$ \\
\hline $\begin{array}{l}\text { Senna uniflora (P. Mill.) H. S. Irwin } \\
\& \text { Barneby }\end{array}$ & $X$ & - & $\mathrm{H}$ & $\mathrm{N}$ \\
\hline \multicolumn{5}{|l|}{ Lauraceae } \\
\hline $\begin{array}{l}\text { Endlicheria paniculata (Spreng.) J. } \\
\text { F. Macbr. }\end{array}$ & - & $\mathrm{X}$ & A & $\mathrm{N}$ \\
\hline \multicolumn{5}{|l|}{ Malvaceae } \\
\hline Sida cordifolia $\mathrm{L}$. & $X$ & - & $\mathrm{H}$ & $\mathrm{N}$ \\
\hline
\end{tabular}

Em que: $\mathrm{Ab}=$ arbustivo; $\mathrm{C}=$ cacto; $\mathrm{H}=$ herbáceo; $\mathrm{N}=$ não ameaçada; $\mathrm{S}=$ ameaçada.
Tabela 4. Densidade relativa da regeneração natural por classe de altura no remanescente florestal em Barrentas (B) no Rio Grande do Norte e Passagem Rasa (PR) na Paraíba.

Table 4. Relative density of natural regeneration by height class in the forest remnant in Barrentas (B) in Rio Grande do Norte State and Passagem Rasa (PR) in Paraíba State.

\begin{tabular}{|c|c|c|c|c|}
\hline \multirow[b]{2}{*}{ Espécies/classes } & \multicolumn{2}{|c|}{ Classe 1} & \multicolumn{2}{|c|}{ Classe 2} \\
\hline & Barrentas & $\begin{array}{c}\text { Passagem } \\
\text { Rasa }\end{array}$ & Barrentas & $\begin{array}{c}\text { Passagem } \\
\text { Rasa } \\
\end{array}$ \\
\hline Acalypha multicaulis & - & 23,0 & - & 22,2 \\
\hline $\begin{array}{l}\text { Aspidosperma } \\
\text { pyrifolium }\end{array}$ & 18,6 & 3,5 & 6,20 & 2,6 \\
\hline Bauhinia cheilantha & - & 0,9 & - & - \\
\hline $\begin{array}{l}\text { Cnidoscolus } \\
\text { quercifolius }\end{array}$ & 2,3 & - & - & - \\
\hline Cordia leucocephala & - & 30,09 & - & 13,7 \\
\hline Croton sp. & - & - & - & - \\
\hline Croton blanchetianus & 32,6 & 29,2 & 13,1 & 36,7 \\
\hline Croton campestris & - & - & - & - \\
\hline $\begin{array}{l}\text { Endlicheria } \\
\text { paniculata }\end{array}$ & - & 04,4 & - & 8,5 \\
\hline Jatropha mollissima & - & 0,9 & 0,7 & 3,4 \\
\hline Mimosa adenophylla & - & - & 1,38 & - \\
\hline $\begin{array}{l}\text { Mimosa } \\
\text { ophthalmocentra }\end{array}$ & - & 2,7 & - & 9,4 \\
\hline Mimosa tenuiflora & - & - & 3,5 & - \\
\hline $\begin{array}{l}\text { Pilosocereus } \\
\text { gounellei }\end{array}$ & 02,3 & - & 42,8 & - \\
\hline Piptadenia stipulacea & - & - & 2,8 & - \\
\hline $\begin{array}{l}\text { Poincianella } \\
\text { pyramidalis }\end{array}$ & 32,6 & 5,3 & 24,8 & 3,4 \\
\hline Sapium glandulosum & - & - & - & - \\
\hline Senna uniflora & 2,3 & - & - & - \\
\hline Sida cordifolia & 9,3 & - & 4,8 & - \\
\hline
\end{tabular}

Nota 1: Valores zerados não foram representativos ao nível de arredondamento considerado. Classe $1=0,5 \mathrm{~m} \leq$ altura $\leq 1,0 \mathrm{~m}$ e Classe $2=$ altura $>1 \mathrm{~m}$

Croton blanchetianus, Pilosocereus gounellei e Poincianella pyramidalis em Barrentas, e Acalypha multicaulis, Croton blanchetianus e Cordia leucocephala em Passagem Rasa, foram as espécies com maior expressividade na regeneração natural. Aspidosperma pyrifolium, Croton blanchetianus, Jatropha molissima e Poincianella pyramidalis tiveram indivíduos identificados na regeneração natural nas duas áreas. 


\section{Discussão}

A suficiência amostral foi consistente com outros trabalhos realizados em área de Caatinga, pois a partir dos $4.000 \mathrm{~m}^{2}$ Guedes et al. (2012) também conseguiram uma amostragem satisfatória. Araújo et al. (2010) amostraram $9.000 \mathrm{~m}^{2}$ para três áreas de estudo, porém verificaram suficiência amostral a partir de $4.200 \mathrm{~m}^{2}$, enquanto Alves et al. (2013) aos $4.400 \mathrm{~m}^{2}$.

O número de famílias, gêneros e espécies em Barrentas foi inferior ao observado por Guedes et al. (2012) em remanescente de Caatinga na Fazenda Tamanduá no interior da Paraíba, onde inventariaram 21 espécies, 21 gêneros e 11 famílias e, por Santana et al. (2009) na Estação Ecológica do rio Grande do Norte, que amostraram 22 espécies, 20 gêneros e 12 famílias. As duas áreas (Fazenda Tamanduá e Estação Ecológica) não sofrem pressão sobre os recursos florestais, como é o caso de Barrentas. Além do histórico de perturbação, vale destacar que as condições edafoclimáticas são distintas, o que influencia diretamente a estrutura, composição florística e distribuição espacial das espécies e indivíduos. Amorim et al. (2005) citam que a Caatinga apresenta grande variação fisionômica, principalmente quanto à densidade e ao porte das plantas. Mudanças em escala local, a poucas dezenas de metros, são facilmente reconhecíveis e geralmente ligadas a uma alteração ambiental claramente identificável. É o caso do maior porte das plantas nos vales e do menor sobre lajedos e solos rasos, em consequência da disponibilidade hídrica diferente.

Apesar da riqueza de famílias, gêneros e espécies ter sido maior em Passagem Rasa do que em Barrentas, ainda foi inferior à observada por Oliveira et al. (2009), em quatro áreas serranas do Cariri Paraibano (de 13 a 17 famílias, de 21 a 34 gêneros, e de 29 a 37 espécies). Essas diferenças na riqueza de famílias, gêneros e espécies entre as áreas pressupõe que não há homogeneidade florística entre os remanescentes. Possivelmente, a pressão antrópica exercida parece influenciar na estrutura da vegetação, conforme cita Holanda et al. (2015) ao estudar a estrutura da vegetação em remanescentes de caatinga com diferentes históricos de perturbação, onde observaram uma menor diversidade florística e estrutural para os indivíduos adultos $(0,80$ nats ind ${ }^{-1}$ e 286 indivíduos) de uma área submetida ao pastejo de ovinos e bovinos, quando comparado a uma área circunvizinha utilizada para extração de lenha (1,50 nats ind ${ }^{-1}$ e 1.155 indivíduos).
As famílias Fabaceae e Euphorbiaceae apresentaram maior riqueza de espécies, ratificando o que outros autores constataram em estudos semelhantes (Barbosa et al., 2007; Santos \& Santos, 2008; Andrade et al., 2009; Oliveira et al., 2009; Santana et al., 2009; Pinheiro et al., 2010; Santos et al., 2011; Guedes et al., 2012).

Os valores de densidade estimados para o estrato adulto ficaram bem próximos nas duas áreas, inferindo-se que o estado de conservação dos remanescentes é semelhante. Esses resultados são semelhantes aos encontrados por Fabricante \& Andrade (2007) em Seridó Paraibano, com 3.130 a 3.250 ind ha ${ }^{-1}$ e por Barbosa et al. (2007) em áreas de serra no Cariri Paraibano (3.010 ind ha ${ }^{-1}$ ). Porém, essa característica não é comum para todos os remanescentes de Caatinga. Santana et al. (2009) registraram 4.080 ind $\mathrm{ha}^{-1}$ no Seridó Potiguar e Oliveira et al. (2009) 4.540 ind ha-1 em áreas de serra no Cariri Paraibano. Valores muito inferiores foram encontrados por Guedes et al. (2012) no sertão da Paraíba (1.622 ind $\mathrm{ha}^{-1}$ ) por Dias et al. (2014) na região de Mossoró, RN (844 ind ha-1) e por Santos et al. (2011) no norte de Minas Gerais em ambiente de transição Caatinga/Cerrado (1.652 ind ha ${ }^{-1}$ ).

As áreas de estudo apresentaram índices de diversidade mais baixos que em outras Caatingas protegidas (espécies lenhosas), conforme avaliaram Oliveira et al. (2009) ( $H^{\prime}$ de 2,52 a 2,35), Santos et al. (2011) e Alves et al. (2013) (2,94 e 2,96 respectivamente), e Guedes et al. (2012) $(2,54)$.

Constatou-se que as áreas de estudo na Depressão Sertaneja (Barrentas) e no Planalto da Borborema (Passagem Rasa) apresentaram baixa similaridade florística para o componente adulto $(S J=0,19)$ e regenerante $(S J=0,21)$. Em princípio, esse resultado está relacionado às questões edafoclimáticas, tendo em vista que o histórico de perturbação é parecido. Não é rara a variação fisionômica de fragmentos florestais em diferentes tipologias, conforme atestaram Hermuche \& Felfili (2011). Outras variáveis ambientais podem resultar em baixa similaridade florística, como por exemplo diferentes tipos de solos. Pinheiro et al. (2010) verificaram baixa similaridade em áreas de Caatinga quando compararam áreas sobre solos cristalinos e áreas sobre solos sedimentares, embora o estudo tenha sido efetuado numa mesma unidade de relevo (Depressão Sertaneja).

Outros fatores bióticos e abióticos, além de atividades antrópicas, podem influenciar e até comprometer os 
processos dinâmicos da regeneração natural. Esses efeitos negativos podem ser mais críticos em ambientes com distribuição irregular de chuvas, seja no tempo e/ou no espaço, acarretando baixa germinação de sementes ou rebrota de tocos e/ou raízes. Esses resultados foram relatados por Fabricante \& Andrade (2007), que encontraram baixa densidade no estrato regenerante da Caatinga no Seridó Paraibano (2.822 ind ha ${ }^{-1}$ ) e Alves et al. (2010) e Holanda et al. (2015), ao estimarem uma densidade de 4.272 ind ha ${ }^{-1}$ e, 4.000 e 3.066 ind ha $^{-1}$, respectivamente, em uma área de Caatinga no Sertão Paraibano.

Além da baixa similaridade observada para o estrato adulto e regenerante, os remanescentes também apresentam diferenças estruturais, quando comparadas às densidades e dominâncias relativas. É válido ressaltar que outros pesquisadores, a exemplo de Rodal et al. (2008), também perceberam esse padrão distinto de distribuição para as espécies da Caatinga.

\section{Conclusão}

Embora inseridos numa região com índice de aridez elevado, é evidente que fatores abióticos interferem diretamente na composição florística e estrutural. Há fortes indícios que fatores edafoclimáticos associados ao nível de perturbação nas áreas comprometem a riqueza, a diversidade, a similaridade florística e a estrutura horizontal das diferentes comunidades.

\section{Referências}

Ana. Agência Nacional de Águas (Brasil). Governo do Estado do Rio Grande do Norte. Programa de Desenvolvimento Sustentável e Convivência com o Semi-Árido Potiguar: relatório de avaliação ambiental. Natal, 2005. 132 p.

Alves, A. R. et al. Análise da estrutura vegetacional em uma área de Caatinga no município de Bom Jesus, Piauí. Revista Caatinga, v. 26, n. 4, p. 99-106, 2013.

Alves, L. S. et al. Regeneração natural em uma área de caatinga situada no município de Pombal-PB. Revista Verde de Agroecologia e Desenvolvimento Sustentável, v. 5, n. 2, 2010.

Amorim, I. L. et al. Flora e estrutura da vegetação arbustivo-arbórea de uma área de Caatingado Seridó, RN, Brasil. Acta Botânica Brasílica, v. 19, n. 3, p. 615-623, 2005.

Andrade, M. V. M. et al. Levantamento florístico e estrutura fitossociológica do estrato herbáceo e subarbustivo em áreas de Caatinga no Cariri Paraibano. Revista Caatinga, v. 22, n. 1, p. 229-237, 2009.
APG. The Angiosperm Phylogeny Group. An update of the Angiosperm Phylogeny Group classification for the orders families of flowering plants: APG III. Botanical Journal of the Linnean Society, v. 161, n. 2, p. 105-121, 2009.

Araújo, K. D. et al. Levantamento florístico do estrato arbustivoarbóreo em áreas de Caatinga no Cariri Paraibano. Revista Caatinga, v. 23, n. 1, p. 63-70, 2010.

Barbosa, M. R. V. et al. Vegetação e flora no Cariri Paraibano. Oecologia Brasiliensis, v. 11, n. 3, p. 313-322, 2007. https://doi. org/10.4257/oeco.2007.1103.01.

Bezerra Júnior, J. G. O. \& Silva, N. M. Caracterização geoambiental da microrregião do Seridó Oriental do Rio Grande do Norte. Revista Holos, v. 2, p. 78-91, 2007. https://doi.org/10.15628/holos.2007.102.

Brasil. Ministério do Meio Ambiente. Instrução Normativa $n^{\circ} .6$ de 23 de setembro de 2008. Diário Oficial [da] República Federativa do Brasil, Brasília, DF, p. 75, 24 set. 2008.

Cientec. Consultoria e Desenvolvimento de Sistemas. Mata nativa 2: manual do usuário. Viçosa, MG, 2006. 295 p.

Cunha, A. B. et al. Consumo da lenha na calcinação da gipsita e impactos ambientais no pólo gesseiro da mesorregião do Araripe-PE. Revista de Biologia e Farmácia, v. 2, n. 1, p. 1-21, 2008.

Dias, P. M. S. et al. Levantamento fitossociológico de remanescentes florestais no município de Mossoró-RN. Revista Caatinga, v. 27, n. 4, p. 183-190, 2014.

Fabricante, J. R. \& Andrade, L. A. Análise estrutural de um remanescente de caatinga no Seridó Paraibano. Oecologia Brasiliensis, v. 11, n. 3, p. 341-349, 2007. https://doi.org/10.4257/ oeco.2007.1103.04.

Felfili, J. M. \& Rezende, R. P. Conceitos e métodos em fitossociologia. Brasília, DF: UnB, Departamento de Engenharia Florestal, 2003. 68 p. (Comunicações técnicas florestais).

Ferraz, E. M. N. et al. Composição florística em trechos de vegetação de caatinga e brejo de altitude na região do Vale do Pajeú, Pernambuco. Revista Brasileira de Botânica, v. 21, n. 1, p. 7-15, 1998. https://doi.org/10.1590/S0100-84041998000100002.

Forzza, R. C. et al. (Coord.) Lista de espécies da flora do Brasil. Rio de Janeiro: Jardim Botânico do Rio de Janeiro, 2010. Disponível em: < http://floradobrasil.jbrj.gov.br/2010/> Acesso em: 7 maio 2019.

Gamarra-Rojas, C. F. L. et al. (Ed.). Banco de dados de plantas do Nordeste. Recife, 2010. Checklist das plantas do Nordeste (versão 1.5).

Guedes, R. S. et al. Caracterização florístico-fitossociológica do componente lenhoso de um trecho de caatinga no semiárido paraibano. Revista Caatinga, v. 25, n. 2, p. 99-108, 2012.

Hermuche, P. M. \& Felfili, J. M. Relação entre NDVI e florística em fragmentos de floresta estacional decidual no Vale do Paranã, Goiás. Ciência Florestal, v. 21, n. 1, p. 41-52, 2011. https://doi. org/10.5902/198050982746.

Holanda, A. C. et al. Estrutura da vegetação em remanescentes de caatinga com diferentes históricos de perturbação em Cajazeirinhas (PB). Revista Caatinga, v. 28, n. 4, p. 142-150, 2015. 
IBGE. Mapa de clima do Brasil. Rio de Janeiro: IBGE, 2002. 1 mapa. Escala 1: 5.000.000.

IBGE. Mapa de vegetação do Brasil. Rio de Janeiro, 2004. 1 mapa. Escala 1: 5.000.000.

IBGE. Mapa de unidades de relevo do Brasil. Rio de Janeiro, 2006. 1 mapa. Escala 1: 5.000.000.

Oliveira, P. T. B. et al. Florística e fitossociologia de quatro remanescentes vegetacionais em áreas de serra no Cariri Paraibano. Revista Caatinga, v. 22, n. 4, p. 169-178, 2009.

Pinheiro, K. et al. Floristic composition of different soil types in a semi-arid region of Brazil. Revista Caatinga, v. 23, n. 2, p. 68-77, 2010.

Rabelo, F. R. C. et al. Dinâmica da vegetação em um fragmento de Mata Atlântica no Nordeste do Brasil. Ciência Florestal, v. 25, n. 1, p. 23-36, 2015. https://doi.org/10.5902/1980509817442.

Rede de Manejo Florestal da Caatinga. Protocolo de medições de parcelas permanentes. Recife: Associação Plantas do Nordeste, 2005. $21 \mathrm{p}$.

Rodal, M. J. N. et al. Levantamento quantitativo das plantas lenhosas em trechos de vegetação de caatinga em Pernambuco. Revista Caatinga, v. 21, n. 3, p. 192-205, 2008.
Santana, J. A. S. et al. Levantamento Florístico e Associação de Espécies na Caatinga da Estação Ecológica do Seridó, Serra Negra do Norte-RN-Brasil. Revista Verde de Agroecologia e Desenvolvimento Sustentável, v. 4, n. 4, 2009. https://doi. org/10.18378/rvads.v4i4.221.

Santos, R. M. et al. Estrutura e florística de um remanescente de Caatinga arbórea em Juvenília, Norte de Minas Gerais, Brasil. Cerne, v. 17, n. 2, p. 247-258, 2011. https://dx.doi.org/10.1590/ S0104-77602011000200013.

Santos, A. M. M. \& Santos, B. A. S. Are the vegetation structure and composition of the shrubby Caatinga free form edge influence? Acta Botânica Brasilica, v. 22, n. 4, p. 1077-1084, 2008. https:// doi.org/10.1590/S0102-33062008000400018.

Silva, J. S. et al. Sinopse das espécies de Croton L. (Euphorbiaceae) no estado de Pernambuco, Brasil. Acta Botânica Brasilica, v. 24, n. 2, p. 441-453, 2010. https://doi.org/10.1590/S0102-33062010000200015.

Silva, F. A. S. \& Azevedo, C. A. V. Assistência estatística. Campina Grande, 2010.

Siqueira Filho, J. A. et al. Guia de Campo de Árvores da Caatinga. Petrolina: Franciscana, 2009. 64 p.

Superintendência de Administração do Meio Ambiente. Atualização do diagnóstico florestal do Estado da Paraíba. João Pessoa, 2004. $268 \mathrm{p}$. 